\title{
Mixed finite volume method for elliptic equations problems
}

\author{
Sun Jia-hui ${ }^{1, \text { a }}$, Pang shi-chun ${ }^{3, c}$, Ma Ming-Juan ${ }^{3, d}$ \\ ${ }^{1}$ No 7855, people's street, Changchun, Jilin Province \\ ${ }^{2}$ No 7855, people's street, Changchun, Jilin Province \\ ${ }^{3}$ No 7855 , people's street, Changchun, Jilin Province
}

Keywords: mixed finite volume method; error estimates; overall space.

Abstract. This paper briefly describes the relevant conclusions of the mixed finite volume method is mainly based on a set and two mesh mixed finite volume method on triangular mesh, also lists the convergence results corresponding format.

\section{Introduction}

A mesh of elliptic equations with mixed finite volume method problem

\subsection{Mixed finite volume method triangular grid}

Consider the second-order elliptic equations:

$$
\left\{\begin{array}{cc}
-\nabla \cdot \kappa \nabla p=f & \text { in } \Omega \\
p=0 & \text { on } \partial \Omega
\end{array}\right.
$$

Introducing a new flow variable: $u:=-\kappa \nabla p$, Then the equation can be rewritten as a first-order partial differential equations below

$$
\left\{\begin{array}{cc}
\nabla \cdot u-f=0 & \text { in } \Omega \\
u+\kappa \nabla p=0 & \text { in } \Omega \\
p=0 & \text { on } \Omega
\end{array}\right.
$$

Mixed finite volume method: Find $\left(u_{h}, p_{h}\right) \in V_{h} \times Y_{h, 0}$ making

$$
\left\{\begin{array}{l}
\left(\nabla \cdot u_{h}-f, \chi_{K}\right)=0 \\
\left(u_{h}+\kappa \nabla p_{h}, \chi_{K}\right)=0
\end{array}\right.
$$

Error estimates:

If the data of the problem is smooth enough data, Making $p \in H^{2} \cap H_{0}{ }^{1}$, and $u(x)=--\kappa \nabla p(x) \in H^{1}(\Omega)^{2}$,

and We assume $\kappa \in W^{1, \infty}$, then there exists a plus constant $\mathrm{C}$ independent of h making

$\left\|p-p_{h}\right\|_{0} \leq C h^{2}\left(|f|_{h}+\|f\|_{0}\right),\left.f\right|_{K} \in H^{1}(K), \forall K \in \Gamma_{h}$

$\left|p-p_{h}\right|_{h} \leq C h\|f\|_{0}$

$\left\|u-u_{h}\right\|_{0} \leq C h\left(\|f\|_{0}+\|u\|_{0}\right)$

$\left|u-u_{h}\right|_{H(d i v ; \Omega)} \leq C h|f|_{h},\left.f\right|_{K} \in H^{1}(K), \forall K \in \Gamma_{h}$

\subsection{Online quadrilateral mixed finite volume method}

Consider a common second order elliptic problem with boundary condition:

$$
-\nabla(\kappa \nabla p)=f(x), \forall x \in \Omega
$$

Weak form:

$$
\left\{\begin{array}{cc}
\left(\kappa^{-1} \underline{u}, v\right)-\sum_{Q \in Q_{h}}\left(\int_{q} \nabla \underline{v} p-\int_{\partial Q} p \underline{v} \cdot n\right)=0, & \forall v \in H_{l o c}(\operatorname{div}) ; \\
\sum_{T \in \Gamma_{h}}\left(\int_{T}-\nabla q \cdot \underline{u}+\int_{\partial T} \underline{u} \cdot n q\right)=(f, q), & \forall q \in H_{l o c}^{1}\left(\Gamma_{h}\right)
\end{array}\right.
$$


We depart from the weak form derived finite volume method, we establish two control volume method: a symmetrical and the other asymmetrical.Defind a bilinear form defined

$$
\begin{aligned}
& \mathrm{A}(v, p ; w, q) \text { on }\left(V_{h}^{\prime}, W_{h}\right) \times\left(\bar{V}_{h}, W_{h}\right) \\
& \mathrm{A}(v, p ; w, q)=a(v, w)+b(w, p)-b(v, q)
\end{aligned}
$$

Then a non-symmetric control volume method:

Order $V_{h}^{\prime}=\widehat{V}_{h}, \bar{V}_{h}=\gamma_{h} \widehat{V}_{h}$, That find

$\widehat{u}_{h} \in \widehat{V}_{h}, p_{h} \in W_{h}$,

$$
\text { Making } \mathrm{A}\left(\hat{u}_{h}, p_{h} ; \gamma_{h} \widehat{w}, q\right)=(f, q)
$$

Another symmetrical control volume method: Order

$$
V_{h}^{\prime}=\bar{V}_{h}=\gamma_{h} \hat{V}_{h} \text {, }
$$

that find $\widehat{u}_{h} \in \widehat{V}_{h}, p_{h} \in W_{h}$, Making

$$
\mathrm{A}\left(\gamma_{h} \widehat{u}_{h}, p_{h} ; \gamma_{h} \widehat{w}, q\right)=(f, q)
$$

Error estimates:

Theorem: Assuming $\mathrm{u}$ is a solution of the weak form factor (5), The $u_{h}$ is the solution of symmetrical method (8) or asymmetric methods (7),then there exits a number $C_{1}>0$ independent of h,making

$\left\|u-u_{h}\right\|_{0}+\left\|p-p_{h}\right\|_{0} \leq C_{1} h\left(\|u\|_{1}+\|p\|_{1}\right)$

\section{Two meshing problems elliptic equations mixed finite volume method}

\subsection{Mixed finite volume method triangular grid}

We still consider the second-order elliptic equations on a plane convex domains:

$\left\{\begin{array}{cc}-\nabla \cdot \kappa \nabla p=f & \text { in } \Omega \\ p=0 & \text { on } \partial \Omega\end{array}\right.$

Which is symmetric positive definite matrix functions and meet the conditions, we introduce a new variable flow, The problem can be transformed into a mixed question follows:

$$
\left\{\begin{array}{cc}
\nabla \cdot u-f=0 & \text { in } \Omega \\
u+\kappa \nabla p=0 & \text { in } \Omega \\
p=0 & \text { on } \Omega
\end{array}\right.
$$

Solving space:

$V=H(\operatorname{div}, \Omega)=\left\{v \in L^{2}(\Omega): \operatorname{div} v \in L^{2}(\Omega)\right\}$, $H^{s}(\operatorname{div}, \Omega)=\left\{v \in L^{2}(\Omega): \operatorname{div} v \in H^{s}(\Omega)\right\}$

In the definition of the lowest order spatial Raviart-Thomas, we use the reference cell on the local space, which is defined as:

$V_{h}(\widehat{Q})=\{\widehat{v}: \widehat{v}=(a+b \widehat{x}, c+d \widehat{x}), a, b, c, d \in R\}$

Then each quadrilateral $Q$ on local spatial unit is defined as:

$V_{h}(Q)=\left\{\left.v\right|_{Q}=\mathrm{P}_{Q} \widehat{v}: \widehat{v} \in V_{h}(\widehat{Q})\right\}$

The whole space:

$V_{h}=\left\{v \in V:\left.v\right|_{Q} \in V_{h}(Q), \forall Q \in Q_{h}\right\}$

If $n_{i}$ is a unit normal vector outside edge of the unit Q, then right $\widehat{v} \in V_{h}(\widehat{Q})$ there, $\left|e_{i}\right| v \cdot n_{i}=\widehat{v} \cdot \widehat{n}_{i}, i=1,2,3,4$ 
Among, $\hat{n}_{i}$ as a unit outside the normal vector on the edge $e_{i}$, The arbitrary $v \in V_{h}$ In any one unit Q edge of the component to a conventional method.

\subsection{Online quadrilateral mixed finite volume method}

We still consider the problem of second order elliptic equations with Neumann boundary condition on the plane convex domains $\Omega$

$$
\left\{\begin{array}{cc}
-\nabla \cdot \kappa \nabla p=f & \text { in } \Omega \\
\kappa \nabla p \cdot n=0 & \text { on } \partial \Omega
\end{array}\right.
$$

Corresponding to the problem (7) standard mixed finite element method is:find $\left(\tilde{u}_{h}, \tilde{p}_{h}\right) \in H_{h} \times L_{h}$ making

$$
\left\{\begin{array}{cl}
\left(\kappa^{-1} \tilde{u}_{h}, v_{h}\right)-\left(\operatorname{div}_{h}, \tilde{p}_{h}\right)=0 & \forall v_{h} \in H_{h} \\
\left(\operatorname{div} \tilde{u}_{h}, q_{h}\right)=\left(f, q_{h}\right) & \forall q_{h} \in L_{h}
\end{array}\right.
$$

Solving space: Trial function space $\mathrm{H}_{h}$ is called the lowest order Raviart-Thomas space, Then define the trial function space $Y_{h}$ Next, define the operator

$$
\begin{aligned}
& \gamma_{h}: H_{h} \rightarrow Y_{h}: \gamma_{h} w_{h}:=\left(\sum_{i, j} u_{h}\left(c_{i+1 / 2, j}\right) \chi_{i+1 / 2, j}, \sum_{i, j} v_{h}\left(c_{i, j+1 / 2}\right) \chi_{i, j+1 / 2}\right) \\
& w_{h}=\left(u_{h}, v_{h}\right)
\end{aligned}
$$

Corresponding variation in the form of volume control: find $u_{h}, p_{h} \in H_{h} \times L_{h}$ making

$$
\left\{\begin{array}{cl}
a\left(u_{h}, \gamma_{h} w_{h}\right)-b\left(\gamma_{h} w_{h}, p_{h}\right)=0 & \forall w_{h} \in H_{h} \\
-c\left(u_{h}, q_{h}\right)=\left(f, q_{h}\right) & \forall q_{h} \in L_{h}
\end{array}\right.
$$

Error estimates:

Theorem: For $\Omega$ regular mesh $Q_{i, j}$, Solution Let the problem (9) is $u_{h}, p_{h}$, And $\mathrm{u}, \mathrm{p}$ is the problem (7) solution, There is nothing to do with an h, However, the constant $\mathrm{C}$ depends on $\left\|\kappa^{-1}\right\|_{\infty},\|u\|_{1},\|\operatorname{divu}\|_{1},\|p\|_{1}$ such

$$
\left\|u-u_{h}\right\|_{H(d i v)}+\left\|p-p_{h}\right\|_{0} \leq C h
$$

\section{References}

[1] So-Hsiang Chou,Panayot S.Vassilevski, A general mixed covolume framework for constructing conservative schemes for elliptic problems, Mathematics of computation.Vol 68, Number227,991-1101.

[2] So-Hsiang Chou,Do Y.Kwak, Mixed covolume methods on rectangular grids for elliptic problems,SIAM J,NUMER.ANAL.Vol.37,No.3,pp.758-771,2000.

[3] So-Hsiang Chou,Do Y..Kwak,Kwang Y.Kim,A general framework for constructing and analyzing mixed finite volume methods on quadrilateral grids:The overlapping covolume case, SIAM J,NUMER.ANAL.Vol.72,No.4,pp.1170-1196.

[4] LI Rong-hua, CHEN Zhong-ying, WU Wei. Generalized Difference Methods for Differential Equations: Numerical Analysis of Minite Volume Element Methods [M].New York; Marcel Dekker,2000.

[5] GAO Guang-hua,WANG Tong-ke. Cubic Superconvergent Finite Method for One-Dimensional Elliptic and Parabolic Equations [J].J Comput AppL Math,2009,233(9):2285-2301

[6]LI Yong-hai. A Generalized Difference Method for the Parabolic Equations [J]. Math Numersin,2002,24(4):487-500. 
[7] WANG Tong-ke. High Accuracy Finite Volume Element Methods for One-Dimensional Second-Order Elliptic and Parabolic Differential Equations [J]. J Numer Methods Comput Appl, 2002, 23(4):264-274.

[8]Plexousakis M ,Zouraris G E. On the Construction and Analysis of High Order Locally Conservative Finite Volume-Type Methods for One-Dimensional Elliptic Problems [J]. SIAM J Numer Anal,2004, 42(3):1226-1260. 